\title{
SWI or T2*: Which MRI Sequence to Use in the Detection of Cerebral Microbleeds? The Karolinska Imaging Dementia Study
}

\author{
S. Shams, J. Martola, L. Cavallin, T. Granberg, M. Shams, P. Aspelin, L.O. Wahlund, and M. Kristoffersen-Wiberg
}

\begin{abstract}
BACKGROUND AND PURPOSE: Cerebral microbleeds are thought to have potentially important clinical implications in dementia and stroke. However, the use of both $\mathrm{T}^{*}$ and SWI MR imaging sequences for microbleed detection has complicated the crosscomparison of study results. We aimed to determine the impact of microbleed sequences on microbleed detection and associated clinical parameters.
\end{abstract}

MATERIALS AND METHODS: Patients from our memory clinic ( $n=246 ; 53 \%$ female; mean age, 62 ) prospectively underwent $3 T$ MR imaging, with conventional thick-section T2*, thick-section SWI, and conventional thin-section SWI. Microbleeds were assessed separately on thick-section SWI, thin-section SWI, and T2* by 3 raters, with varying neuroradiologic experience. Clinical and radiologic parameters from the dementia investigation were analyzed in association with the number of microbleeds in negative binomial regression analyses.

RESULTS: Prevalence and number of microbleeds were higher on thick-/thin-section SWI (20/21\%) compared with T2*(17\%). There was no difference in microbleed prevalence/number between thick- and thin-section SWI. Interrater agreement was excellent for all raters and sequences. Univariate comparisons of clinical parameters between patients with and without microbleeds yielded no difference across sequences. In the regression analysis, only minor differences in clinical associations with the number of microbleeds were noted across sequences.

CONCLUSIONS: Due to the increased detection of microbleeds, we recommend SWI as the sequence of choice in microbleed detection. Microbleeds and their association with clinical parameters are robust to the effects of varying MR imaging sequences, suggesting that comparison of results across studies is possible, despite differing microbleed sequences.

ABBREVIATIONS: CMB = cerebral microbleed; KIDS = Karolinska Imaging Dementia Study; TSWI = thick-section SWI; tSWI = thin-section SWI; WMH = matter hyperintensities

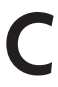

erebral microbleeds (CMBs) have lately become a focus of growing interest. Mainly related to small-vessel disease and seen as a result of hypertensive arteriopathy and cerebral amyloid angiopathy, CMBs have been proposed to have potentially important clinical implications. ${ }^{1-3}$ Theories have proposed that CMBs have a possible important role in the dementia pathophysiology,

Received September 30, 2014; accepted after revision December 16.

From the Department of Clinical Science, Intervention, and Technology, Division of Medical Imaging and Technology (S.S., J.M., L.C., T.G., M.S., P.A., M.K.-W.), and Department of Neurobiology, Care Sciences, and Society (L.O.W.), Karolinska Institutet, Stockholm, Sweden; and Department of Radiology (S.S., J.M., L.C., T.G., M.S., P.A., M.K.-W.) and Division of Clinical Geriatrics (L.O.W.), Karolinska University Hospital, Stockholm, Sweden.

Please address correspondence to Sara Shams, MD, Department of Radiology, Karolinska University Hospital, SE-14186 Stockholm, Sweden; e-mail: sara.shams@ki.se

- Indicates open access to non-subscribers at www.ajnr.org

三 Indicates article with supplemental on-line table.

http://dx.doi.org/10.3174/ajnr.A4248 and additionally, CMBs have shown associations with intracerebral hemorrhage. ${ }^{4}$

Intracerebral hemorrhage and dementia share common characteristics with CMBs. The incidence of cerebral amyloid angiopathy in patients with Alzheimer disease is up to $98 \%,{ }^{5}$ and hypertension has been related to the development of dementia. ${ }^{6}$ Additionally, cerebral amyloid angiopathy and hypertension are the 2 main pathologies behind spontaneous intracerebral hemorrhage, and CMBs are thereby hypothesized to be a possible predictor for intracerebral hemorrhage. ${ }^{7}$

Correct and validated detection is essential to determine and understand $\mathrm{CMBs}$ and their associated clinical implications. CMBs are, due to their microscopic appearance, not visualized on CT or conventional MR imaging. ${ }^{1}$ Detection has, up until now, been with hemosiderin-sensitive sequences, $\mathrm{T}^{\star}$ and SWI. Hemosiderin is a paramagnetic substance, causing inhomogeneity in the magnetic field surrounding the $\mathrm{CMB}$, leading to quick decay of the MR imaging signal, called the "susceptibility effect." T2* is a 
gradient recalled-echo sequence, without a refocusing $180^{\circ}$ radiofrequency pulse, thus making it sensitive to the susceptibility effect. $^{8}$ The SWI sequence, in turn, is a technique that has recently been increasingly incorporated in clinical MRI protocols. SWI maximizes the susceptibility effect by combining a long TE, fully flow-compensated 3D gradient echo, and using both the magnitude and filtered phase information. ${ }^{9,10}$ On sequences sensitive to the susceptibility effect, CMBs are represented by round hypointense dots. Factors of importance in increasing the sensitivity of CMB detection include higher spatial resolution and field and increased TE, with a longer TE increasing the susceptibility effect. ${ }^{3}$ However, this increased sensitivity may come at a cost, possibly contributing to an increased number of false-positive CMBs. Mimics of CMBs include both calcium and iron deposits, flow voids in blood vessels, and cavernomas and partial volume artifacts. ${ }^{3}$ Other reasons for CMBs may be trauma, such as diffuse axonal injury. ${ }^{3}$

While both $\mathrm{T} 2{ }^{\star}$ and SWI have demonstrated good histopathologic correlation, ${ }^{11-13}$ the use of the 2 different $\mathrm{CMB}$ sequences in $\mathrm{CMB}$ detection has complicated the comparison of results across studies. SWI has been shown to increase the number of CMBs detected, ${ }^{3,14,15}$ and studies using the SWI sequence rather than $\mathrm{T}^{*}$ show a higher number and, in some cases, prevalence of CMBs. ${ }^{14,16,17}$ The conventional SWI sequence has a thinner section thickness than the $\mathrm{T} 2{ }^{*}$ sequence. A thin section thickness has been shown to increase $\mathrm{CMB}$ detection ${ }^{15}$; thus, this might contribute to the increased CMB detection seen with SWI. Furthermore, higher field strengths have been shown to increase the number of CMBs detected. ${ }^{15}$ However, whether the increased sensitivity for CMBs with SWI on 3T increases the association of CMBs with clinical parameters remains unknown.

In this study, we aimed to disentangle the effect of sequence from that of section thickness by comparing $\mathrm{CMB}$ detection on the conventional thick-section $\mathrm{T} 2{ }^{*}$, a reconstructed thick-section SWI (TSWI), and the conventional thin-section SWI (tSWI) at 3T. By doing so, we aimed to determine the impact on CMB rating of the different MR imaging sequences and their various effects on clinical associations.

\section{MATERIALS AND METHODS \\ Patients}

This study is part of the Karolinska Imaging Dementia Study (KIDS), a prospective cross-sectional study on the impact of CMBs on dementia. Patients were consecutively and prospectively recruited, and the inclusion criterion for this study was a dementia investigation or follow-up at the memory clinic, Karolinska University Hospital, Stockholm, Sweden. The exclusion criterion for recruitment was any contraindication to undergoing MR imaging. For this study, 246 patients (53\% women; mean age, $62 \pm 10$ years) were recruited and had their brain scan performed on a $3 \mathrm{~T}$ MR imaging scanner with both $\mathrm{T}^{*}$ and SWI added to the general dementia MR imaging protocol. The exclusion criterion for this study during image analysis was insufficient quality of the MR image; however, no patient was excluded for this reason. All patients underwent routine dementia assessment, including medical history; physical, neurologic, and cognitive examinations; laboratory tests; and MR imaging of the brain with CMB sequences. Diagnosis was based on the International Classification of Diseases-10 criteria, by an experienced memory clinic team, consisting of geriatricians, neuropsychologists, neurophysiologists, and neuroradiologists after all aspects had been considered.

Clinical parameters were obtained during the dementia investigation. The presence of hypertension, hyperlipidemia, and diabetes was determined on the basis of prior medical history, diagnosis, and medications. The presence of anticoagulant medication was determined by the routine clinical assessment of each patient's current medications. Each patient was asked about current smoking or alcohol consumption and known hereditary dementia. Body mass index was calculated at the time of dementia investigation and was registered for 177 patients. Cognition was graded by the Mini-Mental State Examination and was registered for 239 patients. The status of hereditary dementia was unknown in 71 patients; current smoking and alcohol consumption status was unknown or missing in 60 and 54 patients, respectively.

Patients included in this study were divided in 4 subgroups, with the following accompanying International Classification of Diseases-10 codes: Alzheimer disease $(n=62)$ : early onset, F00.0 $(n=20)$, late onset, F00.1 $(n=16)$, atypical disease with vascular components, F00.2 $(n=24)$, unspecified Alzheimer disease, F00.9 $(n=2)$; mild cognitive impairment $(n=80)$ : F06.7; subjective cognitive impairment $(n=71)$ : Z03.2A, Z03.3, and R41.8A; other dementias $(n=33)$ : alcohol-related dementia, F10.6, F10.7a $(n=2)$, asymptomatic hereditary dementia $(n=4)$, Z31.5, frontotemporal lobe dementia $(n=4)$, F0.70, Parkinson dementia $(n=6)$, G31.8a, unspecified dementia $(n=10)$, F03.9, vascular dementia $(n=7)$, F01.2, F01.3.

Informed consent was obtained from each patient, and approval was obtained from the regional ethics review board.

\section{MR Imaging Protocol}

All patients $(n=246)$ underwent MR imaging on a 3T Magnetom Trio scanner (Siemens, Erlangen, Germany) with a 12-channel head coil at the radiology department, Karolinska University Hospital, Stockholm, Sweden. For each patient, axial tSWI and $\mathrm{T}^{*}$ sequences and conventional MR imaging sequences, such as T1, T2, and FLAIR, were performed. tSWI was reconstructed to TSWI, by performing a minimum-intensity-projection, by using postprocessing software in the PACS system, making it possible to adjust section thickness and setting it to the same section thickness as that in $\mathrm{T} 2{ }^{\star}$. Parameters for the sequences in this study were the following: tSWI: TE, $20 \mathrm{~ms}$; TR, $28 \mathrm{~ms}$; flip angle, $15^{\circ}$; section thickness, $1.6 \mathrm{~mm}$; intersection gap, $0.3 \mathrm{~mm}$; FOV, $172 \times 230$ $\mathrm{mm}$; in-plane voxel size, $0.8 \times 0.7 \mathrm{~mm}$; total acquisition time, 5 minutes; TSWI: TE, $20 \mathrm{~ms}$; TR, $28 \mathrm{~ms}$; flip angle, $15^{\circ}$; section thickness, $4.0 \mathrm{~mm}$; intersection gap, $1.2 \mathrm{~mm}$; FOV, $172 \times 230$ $\mathrm{mm}$; in-plane voxel size, $0.8 \times 0.7$; T2* ${ }^{*} \mathrm{TE}, 20 \mathrm{~ms}$; TR, $620 \mathrm{~ms}$; flip angle, $20^{\circ}$; section thickness, $4.0 \mathrm{~mm}$; intersection gap, 1.2 $\mathrm{mm}$; FOV, $220 \times 220 \mathrm{~mm}$; in-plane voxel size, $1.1 \times 0.9$; FLAIR: TE, $89 \mathrm{~ms}$; TR, $9000 \mathrm{~ms}$; flip angle, 130'; TI, $2500 \mathrm{~ms}$; section thickness, $4.0 \mathrm{~mm}$; FOV , $199 \times 220 \mathrm{~mm}$; total acquisition time, 5.5 minutes. 


\section{Image Analysis}

For image analysis, 3 raters were chosen on the basis of prior neuroradiologic experience. At the initial time of rating, the raters had the following background experience: Rater 1 was an MD/ $\mathrm{PhD}$ student, with 2 years of training and experience in neuroradiology/MR imaging. Rater 2 had been a neuroradiologist for 10 years. Rater 3 had been a neuroradiologist for 21 years.

All CMB image analysis was performed according to the Microbleed Anatomical Rating Scale, ${ }^{18}$ with the number and topography of $\mathrm{CMBs}$ assessed, as reported before. ${ }^{19}$ Minor modifications were made to the scale to increase the accuracy of the $\mathrm{CMB}$ rating: $\mathrm{CMBs}$ were not rated as probable, only as definitive. Hypointensities in the globus pallidus were not rated, to reduce the risk of calcifications and physiologic iron deposition mimicking CMBs. Furthermore, if the patient had a deep venous anomaly in the vicinity of a CMB, the $\mathrm{CMB}$ was not rated as definitive because deep venous anomalies increase the risk of adjacent cavernomas that, in turn, can mimic a CMB. The T2-weighted images and the $\mathrm{CMB}$ sequences were analyzed simultaneously to better distinguish vessels and flow voids, which might mimic CMBs. The Microbleed Anatomical Rating Scale and the modifications to it were thoroughly discussed by all raters before analysis. A test cohort of patients $(n=20)$ from the original KIDS cohort, with CMBs varyingly on $\mathrm{T} 2{ }^{*}$ or SWI, imaged with the same $3 \mathrm{~T}$ scanner, was assessed by all raters to establish consensus, before initiating the real rating session.

Rater 1 first analyzed all patients $(n=246)$ for CMBs, as part of the original KIDS CMB analysis. ${ }^{19}$ Interrater agreement analysis with Rater 2 was reached on the initial CMB rating, on 50 patients with $\mathrm{CMBs}$ and an additional 50 patients without $\mathrm{CMBs}$, showing an intraclass correlation coefficient of 0.988 for all patients with CMBs, and 0.987 when the 50 patients without CMBs were added to the analysis. ${ }^{19}$ Of all patients, 55 were designated as having CMBs. The 55 patients with CMBs and 20 randomly chosen patients without CMBs were then analyzed by Raters 1, 2, and 3 six months later. The rating procedure was the same for all raters: First $\mathrm{CMBs}$ on the $\mathrm{T} 2{ }^{*}$ sequence were rated continuously on a single day. Three days later CMBs on the tSWI sequence were rated, continuously on a single day. Six months later the TSWI sequence was rated continuously on a single day. The MR images were mixed for each rating session, to randomize the order of $\mathrm{CMB}$ interpretation. The raters were blinded to all patient and clinical data; the CMB sequences were not supposed to be used for rating each other's ratings and their own previous ratings.

White matter hyperintensities (WMH) were graded according to the Fazekas scale, from 0 to 3 (none or single punctate; multiple punctate; early confluent; large confluent). ${ }^{11}$ All images were rated with the rater blinded to clinical data, and other ratings performed. Rater 1 graded all images for WMH on axial FLAIR sequences. Rater 2 graded 50 of the images rated by Rater 1 for interrater agreement analysis. All images rated were randomly chosen with broad representation of the different WMH scores. The weighted $\kappa$ value obtained was 0.94 , which equals excellent agreement.

All radiologic ratings were performed on a PACS workstation with 2 radiologic monitors.

\section{Statistical Analysis}

McNemar and Wilcoxon signed rank tests were used to determine the difference between the prevalence and number of CMBs between sequences; analysis was performed separately between $\mathrm{T} 2{ }^{*}$ and TSWI, T2* and tSWI, and TSWI and tSWI. Intraclass correlation analysis was made on the interrater agreement for CMB detection on the different sequences. $\boldsymbol{\kappa}$-weighted analysis was performed on the interrater agreement on $\mathrm{WMH}$ and is presented under "Image Analysis." The limits for intraclass correlation and $\kappa$ statistics were the following: $0.4-0.6$, moderate agreement; 0.60.8 , good agreement; $>0.8$, excellent agreement. All data were nonparametric; thus, for the univariate analysis of clinical data, $\chi^{2}$ was used for categoric values and Mann-Whitney $U$ tests, for continuous values. Due to the number of patients with zero CMBs, negative binomial regressions were performed to analyze the association between clinical parameters and the number of CMBs. Univariate negative binomial regression analysis was performed with the number of CMBs as a dependent variable and the clinical parameters separately added to the model as independent variables. Subsequently, each regression model was adjusted for age and sex. The univariate comparisons and the regression analyses were performed, as decided a priori, on the median value of CMBs, by all 3 raters. General values given represent the median value on all separate ratings, from all 3 raters for all patients, unless otherwise specified. Likewise, for general values on the prevalence of CMBs, the median prevalence was chosen (ie, if $\geq 2$ raters considered a $\mathrm{CMB}$ present/absent, that became the value representing the median of all raters). The whole cohort $(n=246)$ was included in all statistical analyses. SPSS 22.0 (IBM, Armonk, New York) was used for statistical analysis. All $P$ values presented were post hoc Bonferroni-corrected, and $P<.05$ was set as the threshold of statistical significance.

\section{RESULTS \\ CMB Detection}

The prevalence of CMBs for the whole cohort was $17 \%(n=43)$ on T2* $20 \%(n=50)$ on TSWI, and $21 \%(n=51)$ on tSWI (Table 1). tSWI $(P=.02)$ led to a significant rise in CMB prevalence compared with $\mathrm{T} 2^{*}$, but there was no significant difference between TSWI and T2* and TSWI and tSWI. Similarly, the increase in the number of CMBs detected was significant between $\mathrm{T} 2^{*}$ and TSWI $(P<.001)$ and T2* and tSWI $(P<.001)$, but not between TSWI and tSWI. All raters detected most CMBs on tSWI. Table 1 shows the $\mathrm{CMB}$ detection for the different sequences and raters.

The increase in CMB prevalence on TSWI and tSWI, compared with $\mathrm{T} 2 *$, held true across the different dementia diagnoses (Table 2). There was no difference in prevalence between TSWI and tSWI. The number of CMBs detected increased with TSWI in Alzheimer disease $(P=.006)$ and on tSWI in Alzheimer disease $(P=.006)$ and mild cognitive impairment $(P=.006)$, compared with $\mathrm{T}^{\star}$. There was no significant difference between TSWI and tSWI (Table 2). Table 2 shows the results of CMB detection in the different dementia diagnoses.

\section{Interrater Agreement and Reasons for Disagreement}

Interrater agreement was excellent throughout all sequences and raters (Table 3). Analysis of multiple CMBs (defined as $>1 \mathrm{CMB}$ ) 
still equaled excellent agreement across sequences and raters. The 20 patients without CMBs were classified as not having CMBs by all 3 raters on all sequences. Analyzing the agreement regarding the number of CMBs across all CMB sequences, for the median value of all raters, gave an intraclass correlation value of 0.897 , which equals excellent agreement.

Throughout all ratings, 5 reasons for disagreement were identified. Multiple, pale, and small CMBs were the most frequent reason for disagreement. CMBs close to vessels and obvious CMBs not detected by 1 rater, explained as lack of attention, were other minor reasons for disagreement. Most disagreement was

Table 1: CMBs for each sequence and rater $^{\mathrm{a}}$

\begin{tabular}{|c|c|c|c|c|}
\hline & $\begin{array}{c}\text { All } \\
\text { Raters }\end{array}$ & 1 & 2 & 3 \\
\hline Prevalence of CMBs on T2* (No.) (\%) & $43(17)^{b}$ & $42(17)^{b}$ & $45(18)$ & $38(15)^{\mathrm{b}, \mathrm{c}}$ \\
\hline Prevalence of CMBs on TSWI (No.) (\%) & $50(20)$ & $49(20)$ & 46 (19) & $51(21)^{c}$ \\
\hline Prevalence of CMBs on tSWI (No.) (\%) & $51(21)^{\mathrm{b}}$ & $51(21)^{\mathrm{b}}$ & $50(20)$ & $51(21)^{\mathrm{b}}$ \\
\hline $\mathrm{T}^{*}$ sum CMBs (No.) & $365^{b, c}$ & $373^{b, c}$ & $461^{b}$ & $260^{b, c}$ \\
\hline TSWI sum CMBs (No.) & $528^{c}$ & $570^{c}$ & $450^{d}$ & $576^{c}$ \\
\hline tSWI sum CMBs (No.) & $699^{b}$ & $619^{b}$ & $806^{b, d}$ & $672^{b}$ \\
\hline Times more CMBs on TSWI than $\mathrm{T}^{*}$ & 1.5 & 1.5 & 1.0 & 2.2 \\
\hline Times more CMBs on tSWI than $\mathrm{T}^{*}$ & 1.9 & 1.7 & 1.7 & 2.6 \\
\hline Times more CMBs on tSWI than TSWI & 1.3 & 1.1 & 1.8 & 1.2 \\
\hline
\end{tabular}

${ }^{a} \mathrm{McNemar}$ and Wilcoxon signed rank tests were used to determine the difference in prevalence and sum of CMBs, respectively; analysis was done separately between $\mathrm{T}^{*}$ and TSWI, T2* and TSWI, and TSWI and tSWI. "All Raters" represents the median value of all separate ratings for all patients (ie, not the final values). The whole cohort is included in the analysis $(N=246)$. For "Prevalence," values are given as the number of patients (percentage). All significant values are designated in the Table. All $P$ values have been Bonferroni-corrected.

${ }^{\mathrm{b}} P<.05$ between $\mathrm{T}^{*}$ and $\mathrm{tSWl}$.

c $P<.05$ between $\mathrm{T}^{*}$ and TSWI.

${ }^{\mathrm{d}} P<.05$ between TSWI and $\mathrm{tSWI}$

Table 2: CMBs on $\mathrm{T2}^{*}$, TSWI, and tSWI in the separate dementia diagnoses ${ }^{\mathrm{a}}$

\begin{tabular}{lcccc}
\multicolumn{1}{c}{ Diagnosis } & $\begin{array}{c}\text { AD } \\
(\boldsymbol{n}=62)\end{array}$ & $\begin{array}{c}\text { MCI } \\
(\boldsymbol{n}=80)\end{array}$ & $\begin{array}{c}\text { Other } \\
(\boldsymbol{n}=33)\end{array}$ & $\begin{array}{c}\text { SCI } \\
(\boldsymbol{n}=71)\end{array}$ \\
\hline Prevalence of CMBs on T2* (No.) (\%) & $20(32)$ & $13(16)$ & $6(16)$ & $4(6)$ \\
Prevalence of CMBs on TSWI (No.) (\%) & $22(32)$ & $16(20)$ & $7(22)$ & $5(7)$ \\
Prevalence of CMBs on tSWI (No.) (\%) & $22(35)$ & $16(20)$ & $7(22)$ & $6(8)$ \\
T2* sum CMBs (No.) & $258^{\mathrm{b}, \mathrm{c}}$ & $69^{\mathrm{c}}$ & 47 & 6 \\
TSWI sum CMBs (No.) & $344^{\mathrm{b}}$ & 101 & 73 & 10 \\
tSWI sum CMBs (No.) & $459^{\mathrm{c}}$ & $116^{\mathrm{c}}$ & 79 & 12 \\
Times more CMBs on TSWI than T2* & 1.3 & 1.5 & 1.6 & 1.7 \\
Times more CMBs on tSWI than T2* & 1.8 & 1.7 & 1.7 & 2.0 \\
Times more CMBs on tSWI than TSWI & 1.3 & 1.1 & 1.1 & 1.2 \\
\hline
\end{tabular}

Note:-AD indicates Alzheimer disease; Other, other dementias; $\mathrm{MCl}$, mild cognitive impairment; $\mathrm{SCl}$, subjective cognitive impairment.

${ }^{a}$ Differences in prevalence and sum of CMBs were determined with the McNemar and Wilcoxon signed rank test, respectively; analysis was made between $\mathrm{T2}^{*}$ and $\mathrm{TSWI}, \mathrm{T}^{*}$ and $\mathrm{TSWI}$, and TSWI and tSWI. Ratings represent the median value of all separate ratings for all patients. All significant values are designated in the Table. All $P$ values have been Bonferroni-corrected.

${ }^{\mathrm{b}} \mathrm{P}<.05$ between $\mathrm{T} 2^{\star}$ and TSWI.

${ }^{c} P<.05$ between $\mathrm{T}^{*}$ and $\mathrm{tSWI}$.

Table 3: Interrater agreement for CMBs on $\mathrm{T}^{*}$, TSWI, and $\mathrm{tSWI}{ }^{\mathrm{a}}$

\begin{tabular}{cccccc}
\hline Sequence $^{b}$ & Patients (No.) & All Raters ${ }^{c}$ & Raters 1 and 2 & Raters 1 and 3 & Raters 2 and 3 \\
\hline Analysis 1 & & & & & \\
T2* & 55 & $0.942(0.915-0.961)$ & $0.990(0.981-0.994)$ & $0.858(0.758-0.917)$ & $0.818(0.689-0.894)$ \\
TSWI & 55 & $0.982(0.974-0.988)$ & $0.963(0.942-0.977)$ & $0.994(0.990-0.996)$ & $0.959(0.936-0.974)$ \\
TSWI & 55 & $0.991(0.986-0.994)$ & $0.979(0.964-0.988)$ & $0.997(0.995-0.998)$ & $0.984(0.972-0.991)$ \\
Analysis 2 & & & & & \\
T2* & 25 & $0.959(0.795-0.944)$ & $0.992(0.982-0.996)$ & $0.911(0.798-0.961)$ & $0.882(0.733-0.948)$ \\
TSWI & 37 & $0.984(0.972-0.991)$ & $0.968(0.938-0.984)$ & $0.994(0.988-0.997)$ & $0.963(0.929-0.981)$ \\
TSWI & 33 & $0.991(0.985-0.995)$ & $0.983(0.966-0.992)$ & $0.997(0.994-0.999)$ & $0.983(0.966-0.992)$ \\
\hline
\end{tabular}

${ }^{a}$ Interrater agreement was determined with the intraclass correlation coefficient, and the $95 \%$ confidence intervals are given in parenthesis.

${ }^{\mathrm{b}}$ Analysis was made twice: 1) for all patients $(n=55)$ with CMBs, on the basis of the initial KIDS CMB analysis; 2 ) for all patients with $>1 \mathrm{CMB}$ according to Rater 1 .

c"All Raters" represents analysis among Raters 1, 2, and 3. 
To the best of our knowledge, this is the first study on the impact of MR imaging sequences on CMB detection and accompanying clinical associations in dementia at $3 \mathrm{~T}$. Goos et $\mathrm{al}^{17}$ examined the effect of $\mathrm{T} 2{ }^{*}$, TSWI, and tSWI at $1.5 \mathrm{~T}$, showing a higher number and prevalence of CMBs detected on SWI and no difference in clinical associations among the sequences, after ad-

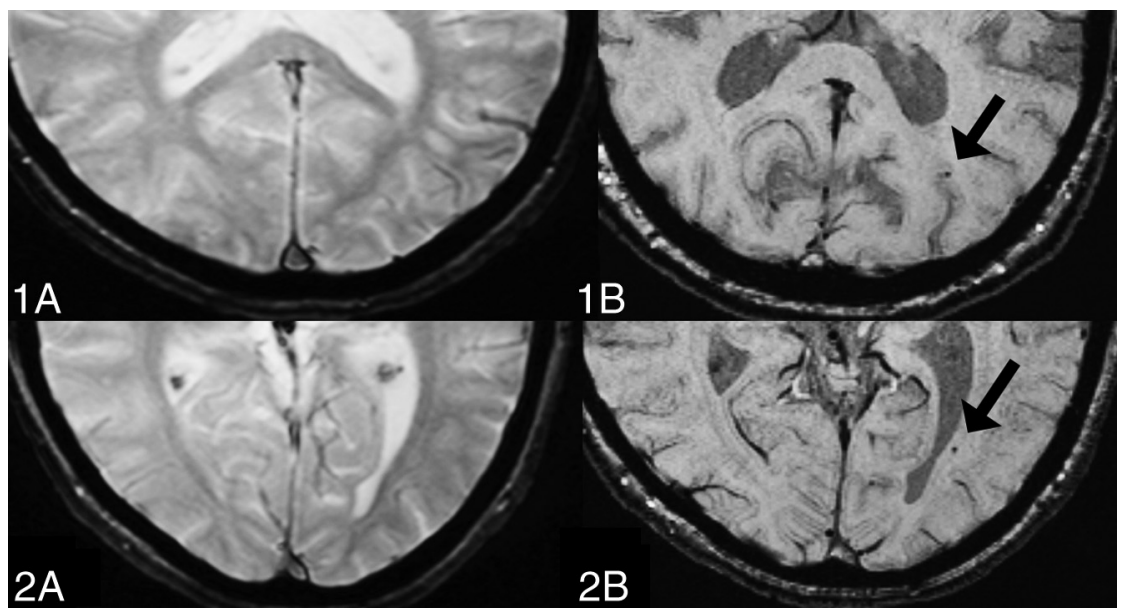

FIG 1. CMBs on tSWI but not T2*. For all images: $A, T 2^{\star}$. $B, \mathrm{tSW}$. 1 , Only Rater 1 identified the CMBs on tSWI. 2, Raters 1 and 2 identified the CMBs on tSWI. justment for age and sex. The higher prevalence and number of CMBs detected with SWI are in accordance with our results and suggest that the increase in CMB detection on SWI across $1.5 \mathrm{~T}-3 \mathrm{~T}$ is associated with the intrinsic processing of the SWI sequence. However, compared with Goos et al, we present more clinical associations with a high number of CMBs, even after adjustment for age and sex. This result may be due to our use of a higher field strength, further sharpening the diagnostic accuracy.

Previous studies investigating the impact of MR imaging sequences on $\mathrm{CMB}$ ratings, especially taking clinical parameters into account, are scarce. The prevalence of CMBs has been shown to be around $18 \%$ to $32 \%$ in Alzheimer disease, versus $3 \%$ to $11 \%$ in healthy aging populations. ${ }^{1}$ The number and prevalence of CMBs have been shown to increase on SWI compared with $\mathrm{T}^{*}$. ${ }^{1}$ Regarding interrater agreement, Goos et $\mathrm{al}^{17}$ showed excellent interrater agreement for both $\mathrm{T} 2{ }^{*}$ and SWI, which is in

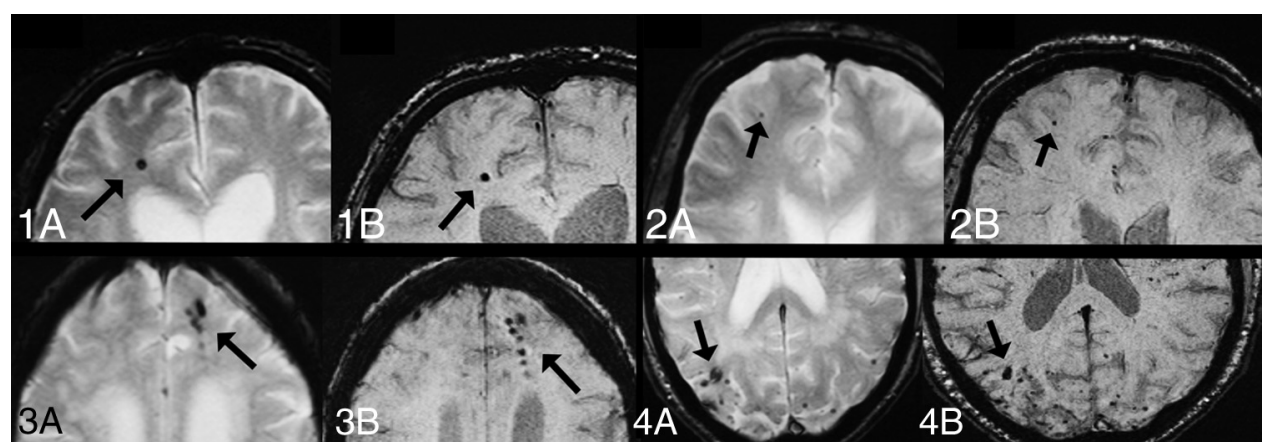

FIG 2. $C M B$ s on both $T 2^{\star}$ and tSWI. For all images: $A, T 2^{*}$. B, tSWI. 1, All raters identified the single $C M B$ on both $T 2^{*}$ and $t S W I$. 2 , The pale $C M B$ on $\mathrm{T2}^{*}$ was only identified by Rater 2 . On tSWI, the CMB was clearly delineated and was identified by all raters. 3 and 4, Disagreement on the exact number of $\mathrm{CMBs}$ occurred on $\mathrm{T}^{*}$. On $\mathrm{tSWI}$, the CMBs are more clearly outlined.

Table 4: Clinical parameters and association with $\mathrm{CMBs}$ depending on sequence ${ }^{\mathrm{a}}$

\begin{tabular}{lcccccc}
\multicolumn{1}{c}{ Clinical Parameters } & $\begin{array}{c}\text { T2* CMB+ } \\
(\boldsymbol{n}=43)\end{array}$ & $\begin{array}{c}\text { T2* CMB- } \\
(\boldsymbol{n}=203)\end{array}$ & $\begin{array}{c}\text { TSWI CMB+ } \\
(\boldsymbol{n}=50)\end{array}$ & $\begin{array}{c}\text { TSWI CMB- } \\
(\boldsymbol{n}=196)\end{array}$ & $\begin{array}{c}\text { tSWI CMB+ } \\
(\boldsymbol{n}=51)\end{array}$ & $\begin{array}{c}\text { tSWI CMB- } \\
(\boldsymbol{n}=195)\end{array}$ \\
\hline Women (No.) (\%) & $19(44)$ & $113(56)$ & $22(44)$ & $110(56)$ & $22(43)$ & $109(56)$ \\
Age (yr) (mean) (SD) & $68(11)^{\mathrm{b}}$ & $63(1)^{\mathrm{b}}$ & $68(11)^{\mathrm{b}}$ & $63(10)^{\mathrm{b}}$ & $68(11)$ & $63(10)$ \\
Hypertension (No.) (\%) & $20(47)$ & $74(37)$ & $23(46)$ & $71(36)$ & $24(47)$ & $70(36)$ \\
Hyperlipidemia (No.) (\%) & $6(14)$ & $31(16)$ & $8(16)$ & $29(15)$ & $9(18)$ & $28(15)$ \\
Diabetes (No.) (\%) & $7(16)$ & $21(10)$ & $8(16)$ & $20(10)$ & $7(14)$ & $21(11)$ \\
Current smoking (No.) (\%) & $4(12)$ & $35(23)$ & $6(15)$ & $33(22)$ & $6(15)$ & $33(23)$ \\
Alcohol (No.) (\%) & $30(91)$ & $131(82)$ & $35(90)$ & $126(82)$ & $36(92)$ & $125(82)$ \\
Heredity (No.) (\%) & $21(72)$ & $82(56)$ & $23(67)$ & $80(57)$ & $23(68)$ & $80(57)$ \\
Anticoagulant medication (No.) (\%) & $11(26)$ & $36(18)$ & $14(28)$ & $33(17)$ & $13(26)$ & $34(18)$ \\
MMSE (mean) (SD) & $25(4)$ & $25(5)$ & $25(4)$ & $25(5)$ & $25(4)$ & $25(5)$ \\
BMI (mean) (SD) & $27(5)$ & $25(3)$ & $25(4)$ & $27(5)$ & $25(4)$ & $27(5)$ \\
WMH $\geq 1$ (No.) (\%) & $38(88)^{\mathrm{b}}$ & $138(68)^{\mathrm{b}}$ & $45(90)^{\mathrm{b}}$ & $131(74)^{\mathrm{b}}$ & $45(88)^{\mathrm{b}}$ & $130(67)^{\mathrm{b}}$ \\
WMH $\geq 2$ (No.) (\%) & $19(44)^{\mathrm{c}}$ & $20(10)^{\mathrm{c}}$ & $21(42)^{\mathrm{c}}$ & $18(9)^{\mathrm{c}}$ & $21(41)^{\mathrm{c}}$ & $18(9)^{\mathrm{c}}$ \\
WMH = 3 (No.) (\%) & $12(28)^{\mathrm{c}}$ & $8(4)^{\mathrm{c}}$ & $14(28)^{\mathrm{c}}$ & $6(3)^{\mathrm{c}}$ & $14(28)^{\mathrm{c}}$ & $6(3)^{\mathrm{c}}$ \\
\hline
\end{tabular}

Note:-MMSE indicates Mini-Mental State Examination; BMI, body mass index; CMB+, patients with CMBs; CMB-, patients without CMBs.

${ }^{a} \chi^{2}$ and Mann-Whitney $U$ tests were done to determine in-sequence differences; significance testing was only done within sequences, and significant values are designated in the Table. All analyses were made on the basis of the median rating value of all raters on all patients. All $P$ values have been Bonferroni-corrected.

${ }^{\mathrm{b}} \mathrm{P}<.05$.

${ }^{c} P<.001$ 
line with findings in our study, whereas Cheng et $\mathrm{al}^{14}$ showed better agreement for SWI. We also noted that our CMB ratings differed slightly between Raters $1 / 3$ and 2 . In contrast to Raters 1 and 3, Rater 2 detected fewer CMBs on TSWI than on tSWI. This finding may be due to the thinner section thickness of the tSWI, facilitating $\mathrm{CMB}$ detection.

We demonstrate that TSWI and tSWI both increase CMB detection, compared with $\mathrm{T} 2{ }^{\star}$. tSWI leads to the largest increase in $\mathrm{CMBs}$, possibly reflecting the impact of section thickness on $\mathrm{CMB}$ detection. ${ }^{15}$ The significant increase in $\mathrm{CMB}$ detection with both TSWI and tSWI is probably due to the innate properties of the SWI sequence. Furthermore, we show that TSWI and tSWI increase the prevalence/number of CMBs, with a minor impact on clinical associations, compared with $\mathrm{T} 2{ }^{\star}$. This finding is of importance for future studies on CMBs and for the interpretation of studies regarding CMBs. Our results suggest that studies with T2* and/or SWI are comparable in clinical associations, implying that the exact number of CMBs may not be of major importance. However, further research is needed to outline the importance of the exact numbers of CMBs, especially in clinical routine neuroradiologic analysis, and until then, the most sensitive sequence in CMB detection, the SWI, is recommended. Patients with multiple CMBs have been shown to demonstrate lower levels of CSF amyloid $\beta 42$ levels compared with patients without CMBs; thus, differentiating patients with single and multiple CMBs with the SWI sequence may provide additional information on the CSF biomarker level of patients. ${ }^{20}$ The advantage of higher sensitivity may further contribute to increasing the power of studies, with possibly fewer subjects needed for analysis. Using SWI compared with $\mathrm{T} 2 *$ is of additional benefit due to its shorter acquisition time on our scanner, though the time difference when compared with $\mathrm{T} 2 *$ was minor.

In addition, we corroborate the known relationship between CMBs and small-vessel disease, by showing an increase in the number of CMBs with increased WMH. Most interesting, other further associations with CMBs, such as alcohol, smoking, and hereditary dementia, were discovered when considering the number of CMBs. This might imply the multifactorial existence of CMBs. The inverse relationship of a high number of CMBs with anticoagulants is surprising and warrants further research.

The strengths of our study include a large cohort, raters with variable neuroradiologic experience, and the use of rating scales for radiologic analyses, contributing to the generalizability of our results. In addition, we compared $\mathrm{T} 2{ }^{*}$ and TSWI with the same section parameters, and tSWI; this comparison makes it possible to further disentangle the effects of sequence from that of section thickness. For additional clarification, $\mathrm{T} 2{ }^{\star}$ with thinsection thickness could have been used. The $\mathrm{CMB}$ rating was performed on the SWI and $\mathrm{T} 2{ }^{*}$ sequences only, in accordance with other similar studies, ${ }^{14,17}$ and with the use of the Microbleed Anatomical Rating Scale. ${ }^{18}$ To minimize calcification mimics of CMBs, we excluded CMBs in the globus pallidus from the rating. However, the use of the phase or quantitative susceptibility maps would have provided additional information and could have helped in differentiating calcifications from CMBs. ${ }^{9,10}$

\section{CONCLUSIONS}

On the basis of the increased number of CMBs detected, we recommend SWI as the sequence of choice for CMB detection. The difference in $\mathrm{CMB}$ prevalence/numbers between $\mathrm{T}^{*}$ and SWI does, however, not affect the association with clinical parameters, suggesting that studies with $\mathrm{T} 2{ }^{\star}$ and SWI are comparable.

\section{ACKNOWLEDGMENTS}

We would like to thank Helena Forssell and Karin Kjellsdotter for their administrative and technical assistance and Seyed-Mohammad Fereshtehnejad and Xiaozhen Li for their engagement in this project.

\section{REFERENCES}

1. Werring DJ, ed. Cerebral Microbleeds: Pathophysiology to Clinical Practice. Cambridge: Cambridge University Press; 2011

2. Cordonnier C, van der Flier WM. Brain microbleeds and Alzheimer's disease: innocent observation or key player? Brain 2011; 134(pt 2):335-44

3. Greenberg SM, Vernooij MW, Cordonnier C, et al. Cerebral microbleeds: a guide to detection and interpretation. Lancet Neurol 2009;8:165-74

4. Kakar P, Charidimou A, Werring DJ. Cerebral microbleeds: a new dilemma in stroke medicine. JRSM Cardiovasc Dis 2012;1: 2048004012474754

5. Jellinger KA. Alzheimer disease and cerebrovascular pathology: an update. J Neural Transm 2002;109:813-36

6. Skoog I, Lernfelt B, Landahl S, et al. 15-year longitudinal study of blood pressure and dementia. Lancet 1996;347:1141-45

7. Lee SH, Bae HJ, Kwon SJ, et al. Cerebral microbleeds are regionally associated with intracerebral hemorrhage. Neurology 2004;62: $72-76$

8. Chavhan GB, Babyn PS, Thomas B, et al. Principles, techniques, and applications of T2*-based MR imaging and its special applications. Radiographics 2009;29:1433-49

9. Haacke EM, Mittal S, Wu Z, et al. Susceptibility-weighted imaging: technical aspects and clinical applications, part 1. AJNR Am J Neuroradiol 2009;30:19-30

10. Mittal S, Wu Z, Neelavalli J, et al. Susceptibility-weighted imaging: technical aspects and clinical applications, part 2. AJNR Am J Neuroradiol 2009;30:232-52

11. Fazekas F, Kleinert R, Offenbacher H, et al. The morphologic correlate of incidental punctate white matter hyperintensities on MR images. AJNR Am J Neuroradiol 1991;12:915-21

12. Schrag M, McAuley G, Pomakian J, et al. Correlation of hypointensities in susceptibility-weighted images to tissue histology in dementia patients with cerebral amyloid angiopathy: a postmortem MRI study. Acta Neuropathol 2010;119:291-302

13. Shoamanesh A, Kwok CS, Benavente O. Cerebral microbleeds: histopathological correlation of neuroimaging. Cerebrovasc Dis 2011; 32:528-34

14. Cheng AL, Batool S, McCreary CR, et al. Susceptibility-weighted imaging is more reliable than $\mathrm{T} 2^{\star}$-weighted gradient-recalled echo MRI for detecting microbleeds. Stroke 2013;44:2782-86

15. Nandigam RNK, Viswanathan A, Delgado P, et al. MR imaging detection of cerebral microbleeds: effect of susceptibility-weighted imaging, section thickness, and field strength. AJNR Am J Neuroradiol 2009;30:338-43

16. Uetani H, Hirai T, Hashimoto M, et al. Prevalence and topography of small hypointense foci suggesting microbleeds on 3T susceptibility-weighted imaging in various types of dementia. AJNR Am J Neuroradiol 2013;34:984-89

17. Goos JDC, van der Flier WM, Knol DL, et al. Clinical relevance of 
improved microbleed detection by susceptibility-weighted magnetic resonance imaging. Stroke 2011;42:1894-900

18. Gregoire SM, Chaudhary UJ, Brown MM, et al. The Microbleed Anatomical Rating Scale (MARS): reliability of a tool to map brain microbleeds. Neurology 2009;73:1759-66

19. Shams S, Martola J, Granberg T, et al. Cerebral microbleeds: differ- ent prevalence, topography and risk factors depending on dementia diagnosis-the Karolinska Imaging Dementia Study. AJNR Am J Neuroradiol 2015;36:661-66

20. Goos JDC, Kester MI, Barkhof F, et al. Patients with Alzheimer disease with multiple microbleeds: relation with cerebrospinal fluid biomarkers and cognition. Stroke 2009;40:3455-60 